\title{
A genetic regulatory network-based sequencing method for mixed-model assembly lines
}

\author{
Lv, Y. ${ }^{a}$, Zhang, J. ${ }^{a,}{ }^{*}$, Qin, W. ${ }^{a}$

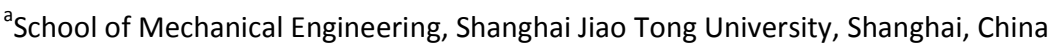

\begin{abstract}
A B S T R A C T
Mixed-model sequencing to minimize work overload at stations is regarded as one of the most concerned optimization problems in assembly lines manufacturing a variety of product models simultaneously. A novel sequencing method based on the genetic regulatory network is proposed to solve this problem. First, genes, gene regulation equations and gene expression procedures are developed in the network based on its similarity with the mixed-model sequencing problem. Each two-state gene represents a binary decision variable of the mathematical model. The gene regulation equations describe decision variable interactions in the constraints and objectives. The gene expression procedure depends on the regulation equations to generate solutions, in which the value of each decision variable is indicated by the expression state of the related gene. Second, regulatory parameter optimization in the regulation equations minimizes the work overload at stations. The effectiveness of the proposed method is validated through experiments consisting of reference instances and industrial instances. The experimental results demonstrate that this method outperforms other methods in large-scale instances.
\end{abstract}

\section{ARTICLE INFO}

Keywords:

Assembly line

Mixed-model sequencing

Work overload

Genetic regulatory network

Genetic algorithm

*Corresponding author: zhangjie@sjtu.edu.cn

(Zhang, J.)

Article history:

Received 11 October 2016

Revised 16 January 2017

Accepted 17 January 2017

(c) 2017 PEI, University of Maribor. All rights reserved.

\section{References}

[1] Moradi, H., Zandieh, M., Mahdavi, I. (2011). Non-dominated ranked genetic algorithm for a multi-objective mixed-model assembly line sequencing problem, International Journal of Production Research, Vol. 49, No. 12, 3479-3499, doi: 10.1080/00207540903433882.

[2] Mukund Nilakantan, J., Ponnambalam, S.G. (2015). Robotic U-shaped assembly line balancing using particle swarm optimization, Engineering Optimization, Vol. 48, No. 2, 231-252, doi: 10.1080/0305215X.2014.998664.

[3] Lian, K., Zhang, C., Gao, L., Shao, X. (2012). A modified colonial competitive algorithm for the mixed-model U-line balancing and sequencing problem, International Journal of Production Research, Vol. 50, No. 18, 5117-5131, doi: $10.1080 / 00207543.2011 .653453$.

[4] Akpinar, S., Bayhan G.M. (2014). Performance evaluation of ant colony optimization-based solution strategies on the mixed-model assembly line balancing problem, Engineering Optimization, Vol. 46, No. 6, 842-862, doi: $\underline{10.1080 / 0305215 X .2013 .806915}$.

[5] Boysen, N., Fliedner, M., Scholl, A. (2009). Production planning of mixed-model assembly lines: Overview and extensions, Production Planning \& Control, Vol. 20, No. 5, 455-471, doi: 10.1080/09537280903011626.

[6] Chan, F.T.S., Wong, T.C., Chan, L.Y. (2009). An evolutionary algorithm for assembly job shop with part sharing, Computers \& Industrial Engineering, Vol. 57, No. 3, 641-651, doi: 10.1016/j.cie.2008.11.017.

[7] Boysen, N., Kiel, M., Scholl, A. (2011). Sequencing mixed-model assembly lines to minimise the number of work overload situations, International Journal of Production Research, Vol. 49, No. 16, 4735-4760, doi: 10.1080/ 00207543.2010 .507607$.

[8] Bautista, J., Alfaro, R., Batalla, C. (2015). Modeling and solving the mixed-model sequencing problem to improve productivity, International Journal of Production Economics, Vol. 161, 83-95, doi: 10.1016/j.ijpe.2014.11.018. 
[9] Boysen, N., Fliedner, M., Scholl, A. (2009). Sequencing mixed-model assembly lines: Survey, classification and model critique, European Journal of Operational Research, Vol. 192, No. 2, 349-373, doi: 10.1016/i.ejor.2007. 09.013.

[10] Wong, T.C., Chan, F.T.S., Chan, L.Y. (2009). A resource-constrained assembly job shop scheduling problem with Lot Streaming technique, Computers \& Industrial Engineering, Vol. 57, No. 3, 983-995, doi: 10.1016/i.cie.2009.04. $\underline{002}$.

[11] McMullen, P.R. (2001). An ant colony optimization approach to addressing a JIT sequencing problem with multiple objectives, Artificial Intelligence in Engineering, Vol. 15, No. 3, 309-317, doi: 10.1016/S0954-1810(01) 00004-8.

[12] Ding, F.-Y., He, J. (2008). A heuristic procedure for the automobile assembly-line sequencing problem considering multiple product options, International Journal of Production Research, Vol. 46, No. 20, 5827-5847, doi: $10.1080 / 00207540701381291$.

[13] Franz, C., Hällgren, E.C., Koberstein A. (2014). Resequencing orders on mixed-model assembly lines: Heuristic approaches to minimise the number of overload situations, International Journal of Production Research, Vol. 52, No. 19, 5823-5840, doi: 10.1080/00207543.2014.918293.

[14] Rahimi-Vahed, A.R., Rabbani, M., Tavakkoli-Moghaddam, R., Torabi, S.A., Jolai, F. (2007). A multi-objective scatter search for a mixed-model assembly line sequencing problem, Advanced Engineering Informatics, Vol. 21, No. 1, 85-99, doi: 10.1016/j.aei.2006.09.007.

[15] Laili, Y., Tao, F., Zhang, L., Sarker, B.R. (2012). A study of optimal allocation of computing resources in cloud manufacturing systems, The International Journal of Advanced Manufacturing Technology, Vol. 63, No. 5, 671-690, doi: 10.1007/s00170-012-3939-0.

[16] Manavizadeh, N., Tavakoli, L., Rabbani, M., Jolai, F. (2013). A multi-objective mixed-model assembly line sequencing problem in order to minimize total costs in a Make-To-Order environment, considering order priority, Journal of Manufacturing Systems, Vol. 32 No. 1, 124-137, doi: 10.1016/j.jmsy.2012.09.001.

[17] Cano-Belmán, J., Ríos-Mercado, R.Z., Bautista, J. (2010). A scatter search based hyper-heuristic for sequencing a mixed-model assembly line, Journal of Heuristics, Vol. 16, No. 6, 749-770, doi: 10.1007/s10732-009-9118-2.

[18] Gujjula, R., Werk, S., Günther, H.-O. (2011). A heuristic based on Vogel's approximation method for sequencing mixed-model assembly lines, International Journal of Production Research, Vol. 49, No. 21, 6451-6468, doi: $\underline{10.1080 / 00207543.2010 .527384 .}$

[19] Cortez, P.M.C., Costa, A.M. (2015). Sequencing mixed-model assembly lines operating with a heterogeneous workforce, International Journal of Production Research, Vol. 53, No. 11, 3419-3432, doi: 10.1080/00207543. 2014.987881.

[20] Akgündüz, O.S., Tunal, S. (2010). An adaptive genetic algorithm approach for the mixed-model assembly line sequencing problem, International Journal of Production Research, Vol. 48, No. 17, 5157-5179, doi: 10.1080/ 00207540903117857.

[21] Shao, X., Wang, B., Rao, Y., Gao, L., Xu, C. (2010). Metaheuristic approaches to sequencing mixed-model fabrication/assembly systems with two objectives, The International Journal of Advanced Manufacturing Technology, Vol. 48, No. 9, 1159-1171, doi: 10.1007/s00170-009-2337-8.

[22] Wang, Y., Liu, J.H. (2010). Chaotic particle swarm optimization for assembly sequence planning, Robotics and Computer-Integrated Manufacturing, Vol. 26, No. 2, 212-222, doi: 10.1016/i.rcim.2009.05.003.

[23] Zhu, Q., Zhang, J. (2011). Ant colony optimisation with elitist ant for sequencing problem in a mixed model assembly line, International Journal of Production Research, Vol. 49, No. 15, 4605-4626, doi: 10.1080/00207543. 2010.493534.

[24] Bautista, J., Cano, A., Alfaro, R. (2012). Modeling and solving a variant of the mixed-model sequencing problem with work overload minimisation and regularity constraints. An application in Nissan's Barcelona Plant, Expert Systems with Applications, Vol. 39, No. 12, 11001-11010, doi: 10.1016/j.eswa.2012.03.024.

[25] Hamzadayi, A., Yildiz, G. (2013). A simulated annealing algorithm based approach for balancing and sequencing of mixed-model U-lines, Computers \& Industrial Engineering, Vol. 66, No. 4, 1070-1084, doi: 10.1016/i.cie.2013. $\underline{08.008 .}$.

[26] Pal, R., Bhattacharya, S., Caglar, M.U. (2012). Robust approaches for genetic regulatory network modeling and intervention: a review of recent advances, IEEE Signal Processing Magazine, Vol. 29, No. 1, 66-76, doi: 10.1109/ MSP.2011.942818.

[27] Balasubramaniam, P., Banu, L.J. (2013). Robust state estimation for discrete-time genetic regulatory network with random delays, Neurocomputing, Vol. 122, 349-369, doi: 10.1016/i.neucom.2013.06.015.

[28] Sun, Y., Feng, G., Cao, J. (2009). Stochastic stability of Markovian switching genetic regulatory networks, Physics Letters A, Vol. 373, No. 18-19, 1646-1652, doi: 10.1016/i.physleta.2009.03.017.

[29] Dilão, R., Muraro, D. (2010). Calibration and validation of a genetic regulatory network model describing the production of the protein Hunchback in Drosophila early development, Comptes Rendus - Biologies, Vol. 333, No. 11-12, 779-788, doi: 10.1016/j.crvi.2010.09.003.

[30] Dörmer, J., Günther, H.-O., Gujjula, R. (2015). Master production scheduling and sequencing at mixed-model assembly lines in the automotive industry, Flexible Services and Manufacturing Journal, Vol. 27, No. 1, 1-29, doi: 10.1007/s10696-013-9173-8.

[31] Bautista, J., Cano, J. (2008). Minimizing work overload in mixed-model assembly lines, International Journal of Production Economics, Vol. 112, No. 1, 177-191, doi: 10.1016/j.ijpe.2006.08.019.

[32] Bautista, J., Cano, A. (2011). Solving mixed model sequencing problem in assembly lines with serial workstations with work overload minimisation and interruption rules, European Journal of Operational Research, Vol. 210, No. 3, 495-513, doi: 10.1016/i.ejor.2010.10.022. 
[33] Tavakkoli-Moghaddam, R., Rahimi-Vahed, A.R. (2006). Multi-criteria sequencing problem for a mixed-model assembly line in a JIT production system, Applied Mathematics and Computation, Vol. 181, No. 2, 1471-1481, doi: 10.1016/j.amc.2006.02.033.

[34] Wong, C.S., Chan, F.T.S., Chung, S.H. (2012). A genetic algorithm approach for production scheduling with mould maintenance consideration, International Journal of Production Research, Vol. 50, No. 20, 5683-5697, doi: $10.1080 / 00207543.2011 .613868$.

[35] Shao, X., Li, X., Gao, L., Zhang, C. (2009). Integration of process planning and scheduling - A modified genetic algorithm-based approach, Computers \& Operations Research, Vol. 36, No. 6, 2082-2096, doi: 10.1016/i.cor.2008. $\underline{07.006}$. 


\section{APEM}

\title{
Genetska regulacijska mreža za določitev zaporedja montaže izdelkov na montažni liniji
}

\author{
Lv, Y. ${ }^{a}$, Zhang, J. ${ }^{\mathrm{a},}{ }^{,}$, Qin, $\mathbf{W} .{ }^{\mathrm{a}}$ \\ ${ }^{a}$ School of Mechanical Engineering, Shanghai Jiao Tong University, Shanghai, China
}

\section{POVZETEK}

Pereč problem pri optimizaciji montažnih linij, na katerih se hkrati izdelujejo različni modeli izdelka predstavlja določitev zaporedja montaže posameznih modelov izdelka $\mathrm{z}$ namenom zmanjšanja preobremenitve delovnih postaj. Predlagan je nov model določitve zaporedja montaže, ki temelji na genetski regulacijski mreži. Najprej so v mreži - na osnovi podobnosti s problemom modela določitve zaporedja montaže različnih modelov izdelka - določeni geni, enačbe za ureditev genov in postopki za izražanje genov. Vsak dvostopenjski gen predstavlja binarno odločitveno spremenljivko matematičnega modela. Enačbe za ureditev genov opisujejo odvisnosti med odločitvenimi spremenljivkami pri omejitvah in ciljih. Postopek za izražanje genov ustvari rešitve v odvisnosti od enačb za ureditev genov, tako da je vrednost vsake odločitvene spremenljivke predstavljena z izraženim stanjem gena. Optimizacija regulacijskega parametra $v$ regulacijskih enačbah nato minimizira preobremenitev dela na postajah. Učinkovitost predlagane metode je potrjena z eksperimenti na referenčnih in industrijskih primerih. Rezultati kažejo, da predlagana metoda prekaša druge metode pri kompleksnejših problemih.

\section{PODATKI O ČLANKU}

Ključne besede:

Montažna linija

Zaporedje različnih modelov

Preobremenitev dela

Genetsko regulativno omrežje

Genetski algoritmi

*Kontaktna oseba: zhangjie@sjtu.edu.cn

(Zhang, J.)

Zgodovina članka:

Prejet 11. oktobra 2016

Popravljen 16. januarja 2017

Sprejet 17. januarja 2017 